Original Article

\title{
Effect of minimizing arm swing while walking on the trunk and gluteal muscles
}

\author{
Min-HeE KIM ${ }^{1)}$, WON-GYU Yoo ${ }^{1)^{*}}$ \\ 1) Department of Physical Therapy, College of Biomedical Science and Engineering, Inje University: \\ 607 Obangdong, Gimhae, Gyeongsangnam-do 621-749, Republic of Korea
}

\begin{abstract}
Purpose] This study used an alternative approach compared with previous studies by investigating the effect of minimizing arm swing while walking on the trunk and gluteal muscles. [Subjects and Methods] The subjects of this study included 13 adult males. [Results] The latissimus dorsi muscle activities during walking with a minimized arm swing were significantly decreased compared with those during walking with a full arm swing. The gluteus medius muscle activities during walking with a minimized arm swing were significantly increased compared with those during walking with a full arm swing. [Conclusion] Therefore, walking with minimized arm swing would be an effective exercise for selective strengthening of the gluteus medius muscle.

Key words: Arm swing, Gluteus medius, Walking
\end{abstract}

(This article was submitted Sep. 6, 2016, and was accepted Sep. 29, 2016)

\section{INTRODUCTION}

In humans, arm swing during gait is a complex and natural movement of the joints ${ }^{1)}$. Stephenson et al. ${ }^{2)}$ suggested that movement of the shoulder joint affects the ankle joint, thereby increasing range of motion and trunk stability ${ }^{2}$. Arm swing during gait also minimizes the increase in angular momentum and energy consumption, and has been reported to increase gait stability ${ }^{3}$. Ford et al. ${ }^{4}$ ) suggested that rotation of the trunk and pelvis decreases when arm swing is limited, and that the gait speed increases due to the increase in cooperation between the upper and lower extremities when there is no such limitation. Consequently, a change in the angle of arm swing, due to increased speed, affects the activeness of the latissimus dorsi, which belongs to the posterior oblique sling system during gait ${ }^{5}$. This means that arm swing during gait can affect movement of the legs, which is consistent with the results of the present study, suggesting that muscle activity of the arms can affect that of the legs as arm swing increases. This study used an alternative approach compared with previous studies by investigating the effect of minimizing arm swing while walking on the trunk and gluteal muscles.

\section{SUBJECTS AND METHODS}

The subjects of this study included 13 adult males who voluntarily consented to participate in the study and had no disease history or any problem with walking. The study purpose and methods were explained to the subjects, who provided informed consent according to the principles of the Declaration of Helsinki before participating. Their average age, height, and weight were $28.5 \pm 4.4$ years, $174.5 \pm 6.6 \mathrm{~cm}$, and $73.8 \pm 5.1 \mathrm{~kg}$, respectively. Surface electromyography (EMG) was used to collect raw EMG data using a wireless system. Surface electrodes were placed on the following muscles: left side of the latissimus dorsi (lateral to the T9 spinous process over the muscle belly), right side of the gluteus medius (proximal third of the distance between the iliac crest and the greater trochanter), and gluteus maximus (the midpoint of a line running from the last sacral vertebrae to the greater trochanter). EMG data were normalized using the maximum voluntary isometric contraction (MVIC) of each muscle, which was measured using the manual muscle test. All participants performed the minimized arm swing and

*Corresponding author. Won-gyu Yoo (E-mail: won7y@inje.ac.kr)

(C2017 The Society of Physical Therapy Science. Published by IPEC Inc.

This is an open-access article distributed under the terms of the Creative Commons Attribution Non-Commercial No Derivatives (by-nc-nd) License $<$ http://creativecommons.org/licenses/by-nc-nd/4.0/>. 
full arm swing while walking on a treadmill for $1 \mathrm{~min}$ at a speed of $2.5 \mathrm{~km} / \mathrm{h}$. The minimization of arm swing while walking involved limiting the arm swing within shoulder flexion and extension by 30 degrees using the guide bar. During the full arm swing while walking, the individual was allowed to swing the arm as much as they wished. Repeated one-way ANOVA was used to analyze the results (SPSS ver. 20.0 statistical package). Significance was defined as $\mathrm{p}<0.05$.

\section{RESULTS}

The latissimus dorsi muscle activities during walking with a minimized arm swing (2.5 $\pm 1.8 \%$ MVIC) were significantly decreased compared with those during walking with a full arm swing ( $4.7 \pm 2.6 \%$ MVIC). The gluteus medius muscle activities during walking with a minimized arm swing (12.0 $\pm 5.3 \%$ MVIC) were significantly increased compared with those during walking with a full arm swing $(9.6 \pm 4.7 \%$ MVIC). The gluteus maximus muscle activities did not differ significantly between the two types of walking $(9.5 \pm 5.2 \%$ and $9.5 \pm 6.4 \%$ MVIC, respectively).

\section{DISCUSSION}

Based on this study, the latissimus dorsi muscle activities during walking with a minimized arm swing were decreased compared with those during walking with a full arm swing. The gluteus medius muscle activities during walking with a minimized arm swing were increased compared with those during walking with a full arm swing. Walking with a minimized arm swing may increase the load laterally and externally by decreasing latissimus dorsi muscle activity as a result of the limited arm swing. Arm swing during gait minimizes the increase in angular momentum. It has been reported that it also increases gait stability ${ }^{2}$. Therefore, the gluteus medius muscle activity was increased to maintain pelvic stability during walking with minimized arm swing. The latissimus dorsi and gluteus maximus muscles are linked obliquely to the posterior spine by the thoracolumbar fascia; this is referred to as the posterior oblique sling system ${ }^{1}$. According to this result, which differs from previous research, the gluteus maximus did not differ significantly between walking with minimized and full arm swing. Instead, it showed a complementary relationship between latissimus dorsi and gluteus medius. The gluteus medius plays an important role in maintaining normal movement patterns of the pelvis and lower limbs, and is considered one of the primary stabilizers in the pelvic area ${ }^{1)}$. Walking with minimized arm swing would increase the gluteus medius activity by decreasing latissimus dorsi activity. Therefore, walking with minimized arm swing would be an effective exercise for selective strengthening of the gluteus medius muscle.

\section{ACKNOWLEDGEMENT}

This work was supported by the 2016 Post-doctoral Research Program of Inje University.

\section{REFERENCES}

1) Wu Y, Li Y, Liu AM, et al.: Effect of active arm swing to local dynamic stability during walking. Hum Mov Sci, 2016, 45: 102-109. [Medline] [CrossRef]

2) Stephenson JL, De Serres SJ, Lamontagne A: The effect of arm movements on the lower limb during gait after a stroke. Gait Posture, 2010, 31: 109-115. [Medline] [CrossRef]

3) Collins SH, Adamczyk PG, Ferris DP, et al.: A simple method for calibrating force plates and force treadmills using an instrumented pole. Gait Posture, 2009, 29: 59-64. [Medline] [CrossRef]

4) Ford MP, Wagenaar RC, Newell KM: Arm constraint and walking in healthy adults. Gait Posture, 2007, 26: 135-141. [Medline] [CrossRef]

5) Conneely M, O’Sullivan K: Gluteus maximus and gluteus medius in pelvic and hip stability: isolation or synergistic activation? Physio Irel, 2008, 29: 6-10. 